\title{
eJRIEPS
}

Ejournal de la recherche sur l'intervention en éducation physique et sport

12 | 2007

Varia

\section{Observation et classification des différents types de récupération du ballon lors de la phase défensive au football}

\section{Eric Duprat et Didier Caty}

\section{(2) OpenEdition \\ 1 Journals}

Édition électronique

URL : https://journals.openedition.org/ejrieps/6168

DOI : 10.4000 /ejrieps. 6168

ISSN : 2105-0821

Éditeur

ELLIADD

Référence électronique

Eric Duprat et Didier Caty, « Observation et classification des différents types de récupération du ballon lors de la phase défensive au football », eJRIEPS [En ligne], 12 | 2007, mis en ligne le 01 juillet 2007, consulté le 28 juin 2021. URL : http://journals.openedition.org/ejrieps/6168 ; DOI : https:// doi.org/10.4000/ejrieps.6168

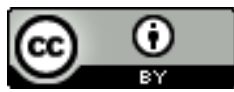

La revue eJRIEPS est mise à disposition selon les termes de la Creative Commons Attribution 4.0 International License. 
Observation et classification des différents types de récupération du ballon lors de la phase défensive au football

\section{Eric Duprat* \& Didier Caty**}

* Département STAPS de I'Université d’Evry, GÉDIAPS de Créteil (Paris XII)

**IUFM de Franche Comté, GRIAPS de Besançon

\section{Résumé}

Ce travail s'inscrit dans les recherches en technologie des APS et concerne plus particulièrement la récupération du ballon en football. Suite à divers constats empiriques, nous souhaitons découvrir des informations susceptibles de fonder notre intervention dans le cadre des apprentissages liés à l'activité. Nos observations basées sur des images de la Coupe du Monde de football de 1998, en France, nous permettent d'extraire des données facilitant la classification des récupérations en quatre types. On distingue celles issues des lois du jeu, celles qui nécessitent l'intervention d'un défenseur, celles qui impliquent plusieurs joueurs et celles qui résultent d'une action du gardien de but. Cette classification, complétée par le positionnement de l'intervention sur l'espace de jeu, nous confirme qu'il existe certaines zones du terrain qui favorisent la récupération et que l'articulation collective des actions est essentielle pour être efficace lors du processus de récupération.

Cette étude s'apparente aux travaux réalisés en technologie des APS (Bouthier \& Durey, 1994) dans le sens où elle s'attache à extraire de la réalité pratique des éléments qui constituent des savoirs théoriques ré-investissables pour le formateur et le joueur. Elle répond aux exigences présentées par David, Bouthier, Marsenach et Durey (1999) qui placent la recherche sur le savoir technique à trois niveaux, «le niveau de la tâche et de l'activité du ou des acteurs ", "le niveau du contexte de la tâche et des ressources des acteurs " et "le niveau du contexte organisationnel de la tâche et la signification sociale des pratiques ». Comme pour Bouthier et Durey (1994), nous visons « une transformation des pratiques » aussi bien pour les joueurs en situation que dans le cadre de l'intervention de l'éducateur, de l'enseignant, du formateur. Dans la lignée des productions de Gréhaigne (1989, 1992, 1994), de Duprat (1996), nous avons développé l'exploration du domaine de la récupération du ballon, en portant notre attention sur les différents types de récupération. Notre étude, porte sur les éléments qui concernent directement l'action 
défensive, au niveau de l'individu et du réseau d'interactions, dans son contexte réel puisque nous saisissons les données à partir des images de matches. Cela nous permet de caractériser certaines constantes événementielles de cette tâche spécifique. Pour ce faire nous nous inspirons de divers auteurs. Pour Dugrand (1989, p. 133) «II n'existe aucune méthode de saisie objective des faits du monde extérieur, pas plus dans la direction du sujet que dans celle de l'activité ». En amont, Wallon (1968, p. 19) pensait déjà que : "L'observation n'est pas un décalque exact et complet de la réalité.... II n'y a pas d'observation sans choix...». Cela implique la détermination de critères observables judicieux et une organisation de l'observation permettant une saisie objectivée des données.

Nous développons une analyse liée à l'aspect topographique en recueillant des données sur tout l'espace de jeu. Notre objectif est de découvrir les zones du terrain qui peuvent rendre les actions plus efficaces quels que soient les facteurs qui entrent en jeu. La première information à saisir concerne donc la position de l'action sur le terrain. Par ailleurs, la notion de supériorité numérique, que l'on retrouve dans les situations de densité, paraît favoriser la reconquête. Nous souhaitons vérifier cette hypothèse en notant si, dans l'action même de la récupération du ballon, un ou plusieurs éléments sont impliqués. L'articulation des actions individuelles trouve ici toute son importance, la simple présence ne suffisant pas. $\mathrm{Si}$, dans certaines zones, le surnombre est réel, il peut aussi exister «artificiellement » grâce aux limites du terrain lorsque le défenseur joue sur son placement, sur l'orientation de son déplacement, et sur celui qu'il cherche à imposer au porteur de balle. Finalement, nous voulons savoir si, dans la majorité des cas, un unique joueur suffit pour s'emparer $\mathrm{du}$ ballon ou bien s'il est nécessaire que plusieurs interviennent dans l'action. II nous faut donc découvrir les caractéristiques des différents « types » d'action rencontrés lors des diverses confrontations traduites par la récupération du ballon. Dans un troisième temps, nous voulons obtenir des données sur les moyens d'action utilisés pour reprendre le ballon, sans aller jusqu'au geste technique, mais en classant les comportements suivant les modalités de l'intervention.

\section{Cadre théorique}

Lorsqu'une équipe est dépossédée du ballon, il lui incombe, dans un premier temps, de ne pas prendre de but, mais surtout de reconquérir celui-ci afin de pouvoir repartir à l'attaque (Hedergott, 1976). Dans tous les cas, "la défense se caractérise par : la récupération du ballon, gêner la progression de l'adversaire vers le but défendu, protéger son but, empêcher de marquer » (Sérafin, 1982, p. 22). Cela nécessite l'utilisation de « techniques 
corporelles » (Vigarello, 1988) par le joueur qui articule ses actions avec celles de ses partenaires proches, en s'inscrivant dans un projet plus global au niveau de l'équipe. Le défenseur doit donc intervenir rapidement pour gêner le porteur du ballon et tenter de s'en emparer lorsque cela est possible. L'objectif est d'empêcher les adversaires de s'approcher du but et de se mettre en position pour tirer et marquer.

Pour de nombreux auteurs, la phase défensive est une affaire d'organisation collective et de gestion des comportements individuels dans un cadre stratégique préalablement établi. "Ainsi on peut dire que l'équipe représente quelque chose de qualitativement nouveau et supérieur. Elle a une valeur supérieure à la somme des valeurs individuelles de chaque joueur, grâce à l'organisation rationnelle " (Téodorescu, 1977, p. 33). Comucci (1977, p. 54) en fait une "base fondamentale " en précisant que : "Toute l'équipe, y compris donc, les demis et les avants sont impliqués dans la défense, quand les adversaires sont en situation d'attaque ». Winterbotton (1952) précisait déjà que plus la défense est compacte plus les chances de réduire les possibilités adverses et d'intercepter le ballon sont grandes et que plus la défense est dispersée plus l'attaque aura de facilité à exploiter les espace libres. Le jeu et sa diversité font appel aux capacités d'intelligence et d'adaptation des joueurs (joueuses). "Les cas que l'on peut rencontrer sont pratiquement infinis. Chaque cas pose son problème, chaque problème apporte sa solution " (Bonizzoni \& Comucci - 1979, p. 167). Tout cela parce qu'au delà des divers facteurs prévisibles et observables (potentiels des joueurs, organisations de base) qui sont déjà créateurs d'incertitudes, il en existe d'autres (stratégie de l'adversaire, conditions météorologiques, état psychologique du moment) qui laissent planer un doute permanent sur l'efficacité du système prévu. II est donc nécessaire de développer les capacités d'adaptation des joueurs et de faire en sorte que la coordination entre les défenseurs réponde aux impératifs liés à la configuration du jeu (Gréhaigne, 1994).

Conforté dans notre idée par les propos de Grosgeorges (1990, p. 69) : "Les observations réalisées en différé vont beaucoup plus loin que les observations traditionnelles. Elles n'ont pas le même objectif, elles tentent d'objectiver les performances, de caractériser des styles de jeu ou de taire des prévisions sur leur évolution ", nous nous sommes référés à la pratique de haut niveau. Notre expérience de joueur puis d'éducateur et de formateur nous a sensibilisé aux problèmes spécifiques rencontrés par le joueur en situation défensive. Le nombre d'échecs observés lors des interventions, durant les matches, nous a conduit à produire une première étude parcellaire (Duprat, 1996) consacrée au tacle, aisément observable grâce à ses caractéristiques. En effet, cette technique se concrétise par un engagement volontaire, 
déterminé, où le joueur essaie de prendre le ballon à l'attaquant, en élargissant son champ d'intervention par une projection du corps au sol. Ce travail, réalisé lors de la phase finale de la Coupe du Monde de 1994 aux USA, nous a permis de constater que, lors du premier tour, le défenseur récupérait seul le ballon dans seulement $3,6 \%$ des cas. Sur l'ensemble des tacles observés (2409) les résultats obtenus montraient que l'équipe récupérait le ballon dans $28 \%$ des cas, pour un taux d'échec avoisinant les $68,5 \%$. Le reste $(3,5 \%)$, difficile à classer, était considéré comme neutre. Plus précisément, nous avons observé que pour les tacles réussis, seulement 12,9\% des interventions se traduisaient par la récupération du ballon par le joueur lui-même, alors que dans 64,3\% c'était un partenaire qui le récupérait. Pour le reste, $22,4 \%$ se traduisaient par une phase statique (touche, coup de pied de but, coup de pied de réparation, coup de pied de coin) et $0,04 \%$ par un but. A l'opposé, les actions négatives se poursuivaient par la continuité du jeu dans $72,2 \%$ des cas avec le déséquilibre défensif qui en découlait, alors que $27,7 \%$ entraînaient une phase statique dont $12,5 \%$ de coups francs et $0,2 \%$ de coups de pied de réparation. Signalons que $29,1 \%$ des avertissements étaient la conséquence d'un tacle (il semblerait que ce taux ait augmenté lors de la dernière Coupe du Monde de 2006) alors que $57,1 \%$ des expulsions résultaient pour une première faute ou une seconde d'un tacle manqué. Le tableau ne serait pas complet si nous omettions de préciser que $32,2 \%$ des buts étaient précédés d'un tacle manqué ou sanctionné.

Nous avons souhaité approfondir notre investigation et avons recherché les travaux déjà réalisés dans le domaine de la récupération du ballon en football. Malgré une exploration étendue, nos investigations ont été peu fructueuses, hormis une recherche effectuée par Lemoine (2005) sur le jeu en déviation qui intègre la notion défensive mais n'en fait pas son centre d'intérêt. Une production de Gréhaigne, Marchal \& Duprat (2001) sur "les conditions de la récupération de la balle en football » aborde néanmoins ce sujet et permet d'apporter un début de piste. Elle propose trois directions pouvant orienter des travaux plus approfondis. La première s'appuie sur les secteurs d'action et d'intervention des joueurs, en fonction de leur positionnement mais surtout de leur vitesse et de leur placement à l'instant $\mathrm{T}$ de la configuration du jeu. Cela permet de présenter les divers cas que l'on peut rencontrer suivant les situations, mais aussi de les comptabiliser durant la rencontre pour faire ressortir les plus efficaces. A ce titre, Gréhaigne avance que la position en barrage est une situation privilégiant la reconquête et que le facteur vitesse est très important. La seconde s'appuie sur le nombre de joueurs engagés dans l'action, ce qui lui permet de constater que la supériorité numérique est un élément favorable à la récupération. Dans un troisième temps, l'auteur a voulu savoir quelles étaient les zones 
privilégiées pour reconquérir le ballon. II fait ressortir qu'il existe, sans comptabiliser les interventions du gardien de but, une zone approximativement placée à douze mètres du but, d'une largeur équivalente aux trois cinquièmes du terrain, répartie équitablement de chaque côté de son axe, qui s'étend jusqu'à environ 40 mètres de la ligne de but, où l'on récupère le plus de ballons. Cette étude, limitée à quatre rencontres de la Coupe du Monde 1994, constitue le premier support de notre travail.

\section{Méthodologie}

\section{1. Etude empirique}

Cette étude technologique consiste à extraire de la réalité, dans notre cas le match, des indicateurs factuels qui permettent de mettre à jour des facteurs favorisant la performance et d'établir des bases théoriques de la pratique. Pour cette seconde observation, nous avons travaillé sur la Coupe du Monde de Football qui s'est déroulée en France, durant la période du mercredi 10 juin au dimanche 12 juillet 1998. Les trente deux équipes qualifiées à l'issue d'un tour préliminaire effectué au sein de chaque confédération continentale (6 dans le Monde) sont regroupées lors de la phase finale. Elles sont réparties, par tirage au sort, en huit groupes de quatre équipes qui se rencontrent sous la forme d'un mini championnat. À l'issue de cette première phase les deux premiers de chaque groupe sont qualifiés pour les huitièmes de finale. À partir de ce stade de la compétition, tous les matches doivent désigner un vainqueur et constituent notre banque de données. A l'origine, les travaux d'analyses réalisés en football s'appuyaient sur un découpage du terrain en quatre zones égales dans la longueur, deux dans la moitié offensive et deux dans la moitié défensive et quatre couloirs équivalents dans la largeur répartis par paires de chaque côté de l'axe central du terrain. Gréhaigne (1992, p. 56), parmi d'autres, fait évoluer la prise de données en divisant l'espace de jeu en huit bandes dans la longueur, réparties de chaque part de la ligne médiane, la bande $A$ se situant près de la cible adverse, alors que la bande $\mathrm{H}$ se trouve plus rapprochée du but défendu. La division sur la largeur s'organise autour de cinq couloirs, dont un axial qui permet de matérialiser le couloir central du jeu considéré comme la colonne vertébrale sur laquelle l'équipe se structure. 


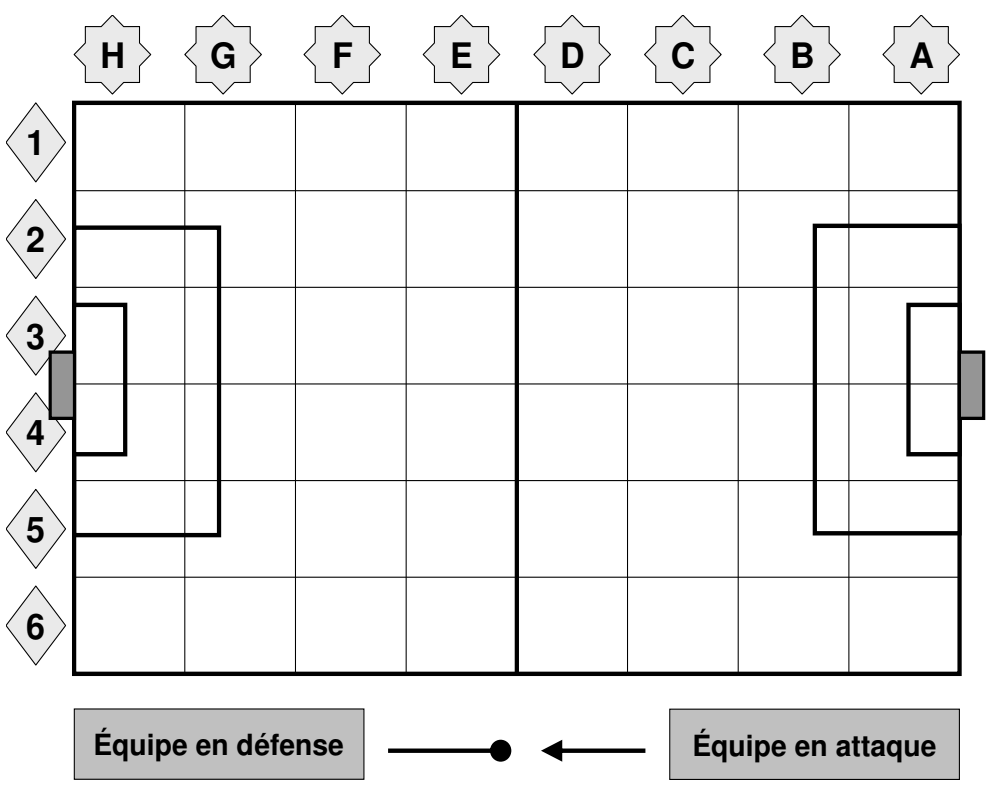

Figure 1. Schéma du quadrillage du terrain en 48 zones pour la saisie

Pour notre part (figure 1), nous choisissons de conserver le même découpage en huit bandes dans la longueur, mais nous optons pour une division en six couloirs sur la largeur afin de bien distinguer la répartition des récupérations de part et d'autre de l'axe longitudinal du terrain. Nous disposons, au départ, du tracé d'un terrain proportionnel aux dimensions réglementaires (cent cinq mètres de long sur soixante-huit de large pour les compétitions internationales), divisé en quarante-huit zones rectangulaires équivalentes, d'environ treize mètres (13,125 m.), sur onze (11,33 m.). Nous notons les bandes en allant de $\mathrm{A}$ à $\mathrm{H}$, sachant que la zone $\mathrm{A}$ se situe près de la cible adverse alors que la $\mathrm{H}$ est la plus rapprochée du but de l'équipe en défense; et les couloirs de 1 à 6 en partant de la gauche, dos au but. Nous choisissons d'observer différents points particuliers pouvant nous donner des informations sur la récupération du ballon, au sens reconquête de l'objet convoité, c'est-à-dire d'une nouvelle possession de balle. Nous estimons que la balle est redevenue la "propriété » d'une équipe lorsque au moins deux joueurs différents la touchent, sans intervention directe d'un adversaire entre temps, ou lorsque le même joueur la maîtrise. Cette maîtrise est réalisée lorsque le joueur effectue au moins deux contacts avec le ballon ou lorsqu'il se retrouve seul au moment de la récupération, même s'il joue en une touche de balle. Nous inscrivons les critères que nous sélectionnons en fonction de la configuration du jeu au moment de la reconquête du ballon par l'équipe en défense. Nous avons testé, au préalable, ces différents repères, ce qui nous a permis d'ajuster et de compléter les diverses caractéristiques de tel ou tel critère. 
En dehors de quelques cas particuliers, nous ne relevons pas d'informations sur l'origine de l'action qui provoque parfois la situation de récupération et qui se trouve très éloignée du point d'arrivée. Nous reviendrons sur cet aspect qui joue indubitablement un rôle important dans la globalité de la situation. Lors de l'observation vidéo, nous disposons un papier calque sur le tracé du terrain découpé en zones. Sur ce calque nous indiquons, pour chaque reconquête de balle, le numéro de l'action à l'endroit où elle se situe sur le terrain, la trajectoire du ballon si cette récupération concerne des joueurs répartis dans deux ou trois zones, et l'implication du gardien de but par un signe distinctif. En parallèle nous notons, pour chaque action, quatre à cinq éléments nous permettant une classification ultérieure. Précisons que quelques rares actions (13) ne peuvent être totalement exploitées, car les conditions de retransmission (ralenti) masquent en partie le déclenchement du mouvement.

\section{2. Informations recueillies}

Les informations recueillies indiquent pour chaque récupération :

- Le numéro de l'action qui suit le déroulement chronologique du jeu dès le coup d'envoi considéré comme une possession de balle. II est encerclé pour une action « simple », et encadré lorsque le gardien de but intervient. L'emplacement du numéro indique, au plus près, en se basant sur l'image et en intégrant l'appréciation des distances, l'endroit en une ou plusieurs étapes de la reconquête.

- La zone où a lieu la récupération que l'on indique à partir de deux éléments qui sont la lettre pour la profondeur ( $\mathrm{A}$ près du but adverse, $\mathrm{H}$ proche de son propre but) et le chiffre pour la largeur, sachant que le couloir 1 est situé le plus à gauche, proche de la ligne de touche, et le 6 le plus à droite près de la ligne de touche opposée.

- Les différents types de récupération (quatre) que nous classons à partir de critères nettement observables :

* les Récupérations Réglementaires (RR), correspondent à l'intervention des arbitres pour le respect des lois du jeux, soit en rapport avec les limites du terrain, la marque et le début des périodes de jeu ; soit dans le cadre des droits des joueurs en attaque et en défense.

* les Récupérations Individuelles (RI), pour lesquelles n'intervient qu'un seul joueur qui prend en charge le ballon sans que les partenaires ne le touchent. Soit le même joueur réalise au minimum deux touches de balle successives, soit il ne réalise qu'un impact mais sans pression d'un adversaire direct, sa liberté d'action est entière. Le fait que l'adversaire évolue dans un espace réduit dû à la densité défensive est signalé mais non pris en compte.

* les Récupérations Collectives (RC), qui nécessitent l'investissement d'au moins deux 
joueurs de la même équipe. II y a donc une prise en charge du ballon en deux étapes (ou trois) qui peuvent se situer dans la même zone ou dans deux, voire trois zones différentes. La supériorité numérique dans l'espace de jeu est précisée dans le relevé, mais non systématique.

* les Récupérations du Gardien de But (GB) qui peuvent être individuelles (Ri) ou collectives (Rc) mais impliquent obligatoirement que ce joueur, au pouvoir exceptionnel (jeu à la main), soit le premier protagoniste de l'action.

Afin d'ajouter des précisions utiles à notre analyse, nous avons constitué, pour chaque «type », diverses catégories que l'on retrouve dans la classification au niveau des « modes d'action ». Ainsi pour les « récupérations réglementaires » $(R R)$ nous avons pu distinguer six cas différents : le coup d'envoi (Engt.), les sorties en touches (TO+), les coups de pied de but $(6 \mathrm{~m})$, les coups francs $(\mathrm{CF}+)$, les situations de hors-jeu $(\mathrm{H}$. jeu) sanctionnées aussi par un coup franc et exceptionnellement les coups de pied de coin $(\mathrm{CR}+)$.

Lorsqu'on explore les différentes situations rencontrées en défense, on observe le plus souvent que l'action défensive s'exerce directement sur un joueur porteur du ballon ou bien sur un adversaire qui tente de se l'approprier. C'est à ce titre que nous distinguons dans nos travaux les situations où le défenseur est en confrontation directe avec le porteur de balle (duel), de celles où le ballon est accessible à tous (conquête). Les autres cas correspondent à des actions lors des trajectoires du ballon traduit en terme d'interception, avec ou sans anticipation, ou bien à des récupérations d'un joueur seul issues d'une erreur adverse provoquée, d'une relance approximative ou d'un dégagement réalisé sous pression.

Pour les « récupérations individuelles » $(\mathrm{RI})$ et les « récupérations collectives » $(\mathrm{RC})$, nous sélectionnons cinq « modes d'action » pour effectuer la catégorisation :

Le Duel: l'adversaire est maître du ballon et s'oppose au défenseur, celui-ci peut être placé en obstacle, barrage, ou bien à la poursuite du porteur.

La Conquête : le ballon n'est maîtrisé par personne, nous sommes sur un «temps de passe », les joueurs évoluant à proximité peuvent s'emparer du ballon, mais il existe une confrontation.

L'Interception: le défenseur intervient lors de la passe, sur la trajectoire qu'il coupe pour s'emparer du ballon en le contrôlant. Cette interception peut avoir lieu, sans distinction, près de la source ou proche du réceptionneur suivant le placement et déplacement du défenseur qui intercepte. 
L'Interception sur anticipation: nous sommes là dans une situation très intéressante, il nous semble, pour l'avenir. Le défenseur, qui intercepte le ballon, a anticipé sur la trajectoire de balle. C'est-à-dire qu'il s'est engagé avant ou au moment de la frappe en réalisant « un pari » sur l'action du passeur. Ce qui distingue cette action de l'interception simple, c'est le mouvement plus engagé, enclenché plus tôt, qui permet d'arriver en avance sur le ballon. La position éloignée ou décalée du défenseur laisse à penser au porteur qu'il bénéficie d'une distance de sécurité dans la passe.

La Récupération d'un " joueur isolé » (seul) : la prise en charge de la balle se réalise alors sans difficulté puisque le défenseur se retrouve seul ou avec une avance confortable pour regagner le ballon, suite à une erreur adverse résultant souvent d'une relance effectuée sous pression ou d'une recherche de la profondeur mal ajustée.

Nous avons observé un mode d'intervention particulier, que nous appelons «piqueballon », qui consiste à laisser l'adversaire contrôler la balle avant d'intervenir pour s'en emparer durant la phase de rééquilibration du joueur.

Pour les « récupérations du gardien de but » (GB) nous avons systématiquement une intervention de ce joueur, au statut particulier, en premier sur le ballon. Soit il le capte en interceptant ou il arrête un tir, soit il le relâche ou le repousse et c'est un partenaire qui le récupère dans un second temps.

De plus, pour l'ensemble des récupérations nous notons les situations où la reconquête résulte d'une action qui s'est «étendue » sur deux, voire trois zones différentes. Cette configuration n'apparaît qu'exceptionnellement lorsqu'il s'agit des « récupérations réglementaires » (3) ou rarement pour les « récupérations individuelles » (16), et se trouve très majoritairement représentées dans les « récupérations collectives 》 (839). Parfois nous ajoutons un élément précisant l'origine de l'action terminée par la récupération.

Pour chaque rencontre et chaque équipe, les données sont informatisées afin de faciliter le traitement. Un programme a été réalisé par François $\mathrm{MÜNCH}$, spécialiste informatique, pour nous permettre de sélectionner les informations suivant certains critères, en fonction des besoins, et de les imprimer. Toutes ces données sont regroupées dans des tableaux récapitulatifs consultables dans la thèse (Duprat, 2005).

\section{Résultats}

Les chiffres bruts, apparus dans les différents tableaux, nous semblent suffisamment «parlants » (Parlebas \& Cyffers, 1992, p. 100) pour que nous puissions réaliser certains constats en nous basant sur les effectifs et les proportions.

3. 1. Présentation des résultats pour l'ensemble des récupérations 
Pour un total de 3460 récupérations du ballon : soit 1734 pour la première série de rencontres concernant les huitièmes de finale et 1726 pour la seconde se rapportant aux quarts, demies finales et finales, nous parvenons à une moyenne d'environ 108 possessions par équipe et par match. La rencontre qui se solde par le plus grand nombre de reconquêtes atteint 127 récupérations (France - Paraguay), alors que le plus faible se situe à 96 (Brésil - Danemark). Nous présentons dans le tableau joint un état des principaux résultats, sachant qu'ils s'articulent autour de la distinction en quatre catégories distinctes qui traduisent soit un arrêt momentané du jeu: « récupérations réglementaires », soit une action dans la continuité du jeu : « récupérations individuelles », « récupérations collectives », « récupérations du gardien de but ».

Tableau I. Répartition des récupérations par type.

\begin{tabular}{|c|c|c|c|}
\hline $\begin{array}{c}\text { Récupérations } \\
\text { Réglementaires }\end{array}$ & $\begin{array}{c}\text { Récupérations } \\
\text { Individuelles }\end{array}$ & $\begin{array}{c}\text { Récupérations } \\
\text { Collectives }\end{array}$ & $\begin{array}{c}\text { Récupérations } \\
\text { du Gardien de } \\
\text { but }\end{array}$ \\
\hline 922 & 928 & 1251 & 346 \\
\hline $26,7 \%$ & $26,9 \%$ & $36,3 \%$ & $10 \%$ \\
\hline
\end{tabular}

Les « RC » sont celles qui rapportent le plus de ballons avec un taux supérieur de près de dix pour cent aux «RR » et « $R I$ », pour lesquelles les effectifs sont très proches. Les récupérations des gardiens de but se limitent à 10\%. Si l'on se penche sur les différences entre les deux groupes de matches que nous distinguons, nous observons des effectifs presque équivalents pour les récupérations réglementaires qui passent de 459 soit 26, \%, à 463 soit 26,9\%; une augmentation sensible des récupérations individuelles de 447 soit $25,9 \%$, à 481 soit $28 \%$; une diminution relative des récupérations collectives de 637 soit $36,8 \%$, à 614 soit $35,7 \%$, et une autre baisse proportionnellement plus importante de 186 soit 10,8\%, à 160 soit 9,3\% pour les interventions des gardiens de but. Dans les deux échantillons de matches, les « récupérations collectives» devancent nettement les « récupérations individuelles » et les « récupérations réglementaires » dans les mêmes proportions, et celles du gardien de but qui avoisinent les $10 \%$.

La répartition de l'ensemble des récupérations (figure 2), à l'exception de celles issues des gardiens de but, nous montre qu'une grande part se situe dans la moitié défensive 85,9\%, pour seulement $14,1 \%$ dans la moitié offensive. Leur positionnement sur le terrain fait apparaître la forme de l'entonnoir traditionnellement présentée. Si l'on précise leur répartition, on constate dans la profondeur deux lignes forces en bande $\mathrm{G}$ et en bande $\mathrm{H}$, 
alors que dans la largeur les récupérations sont concentrées dans l'axe du terrain plus on se rapproche du but.

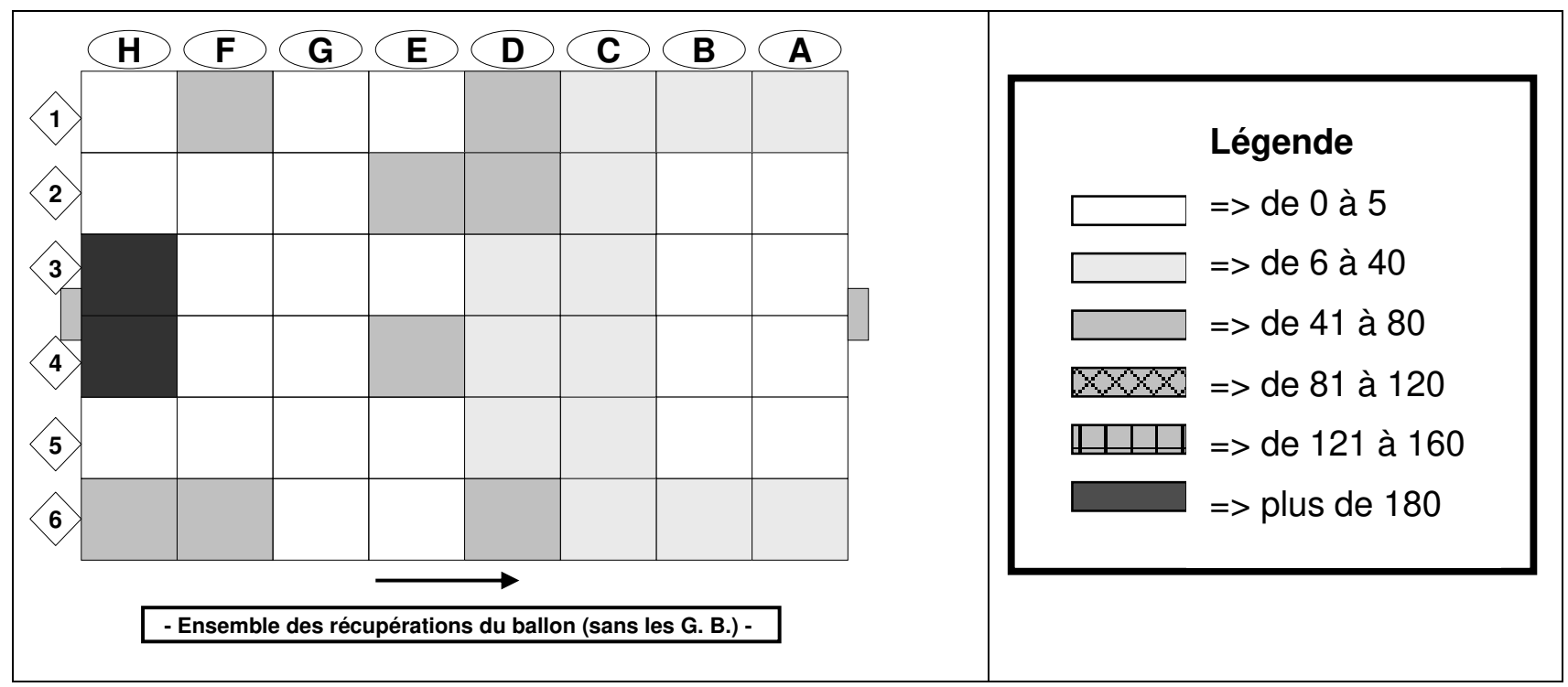

Figures 2 : Répartition des récupérations suivant les « types « (sans les G. B.)

3. 2. Les « récupérations réglementaires 》

Au stade des « récupérations réglementaires » (figure 3), on distingue une répartition sur l'ensemble du terrain avec de nombreuses zones à faibles effectifs. La majorité d'entre elles se trouve à la périphérie de l'espace de jeu, surtout dans la moitié défensive.

Elles confirment l'idée que les limites peuvent être des atouts dans la récupération du ballon. En effet, les coups de pied de but représentent presque 41,6\% des récupérations en $\mathrm{H}$ hors les interventions des gardiens de but, et $14,8 \%$ se situent en dehors des zones $\mathrm{H}-3$ et $\mathrm{H}-4$. A signaler le nombre relativement important de ballons qui sortent en zone $\mathrm{H}$ 5, suite à un jeu profond transversal venant de la droite vers les attaquants. Les touches atteignent $52,6 \%$ de la bande $A, 60 \%$ en B, 22,6 \% en C et encore $17,1 \%$ en D. Leurs proportions dans les cases défensives des couloirs d'ailes obtiennent des valeurs révélatrices de leur importance. Nous pouvons donc considérer les limites de l'espace de jeu comme des partenaires virtuels qui participent à la mise en échec des attaquants. Les zones H-3, 4, G-2, 3, 4, 5, F- 2, 3, 4 et E-4 regroupent l'essentiel des «RG » suite à un hors-jeu et représentent, surtout en $G$ et $F$, une place très importante. Cela confirmerait l'idée qu'il existe une « zone privilégiée » pour utiliser défensivement cette loi. Là encore une règle du jeu s'inscrit comme une aide à l'action défensive. 


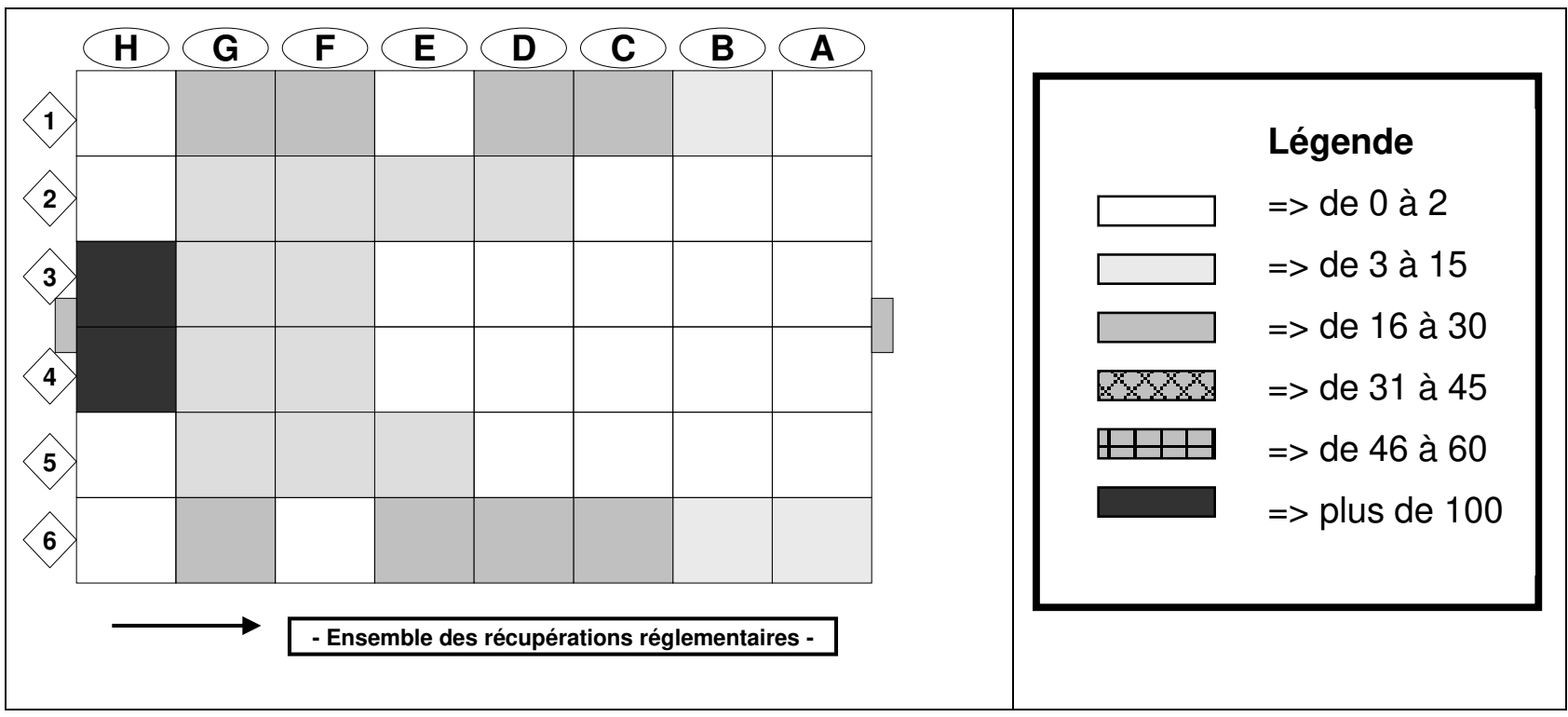

Figure 3. Répartition des récupérations réglementaires

3. 3. Les « récupérations individuelles »

Au niveau des «récupérations individuelles» (figure 4), une majorité d'entre elles se situent assez haut sur le terrain en avant et en arrière de la bande $F$, avec une proportion un peu plus faible sur les couloirs d'ailes, sauf en F-1. Le quadrillage serré des zones F-2, F-3, F-4 et F-5 pourrait laisser à penser qu'il s'agit, en fait, des zones prioritairement utilisées par les attaquants adverses, dont le plus à gauche joue généralement en appui, alors que le droit propose des solutions en profondeur, plutôt vers la droite c'est-à-dire la gauche de la défense.

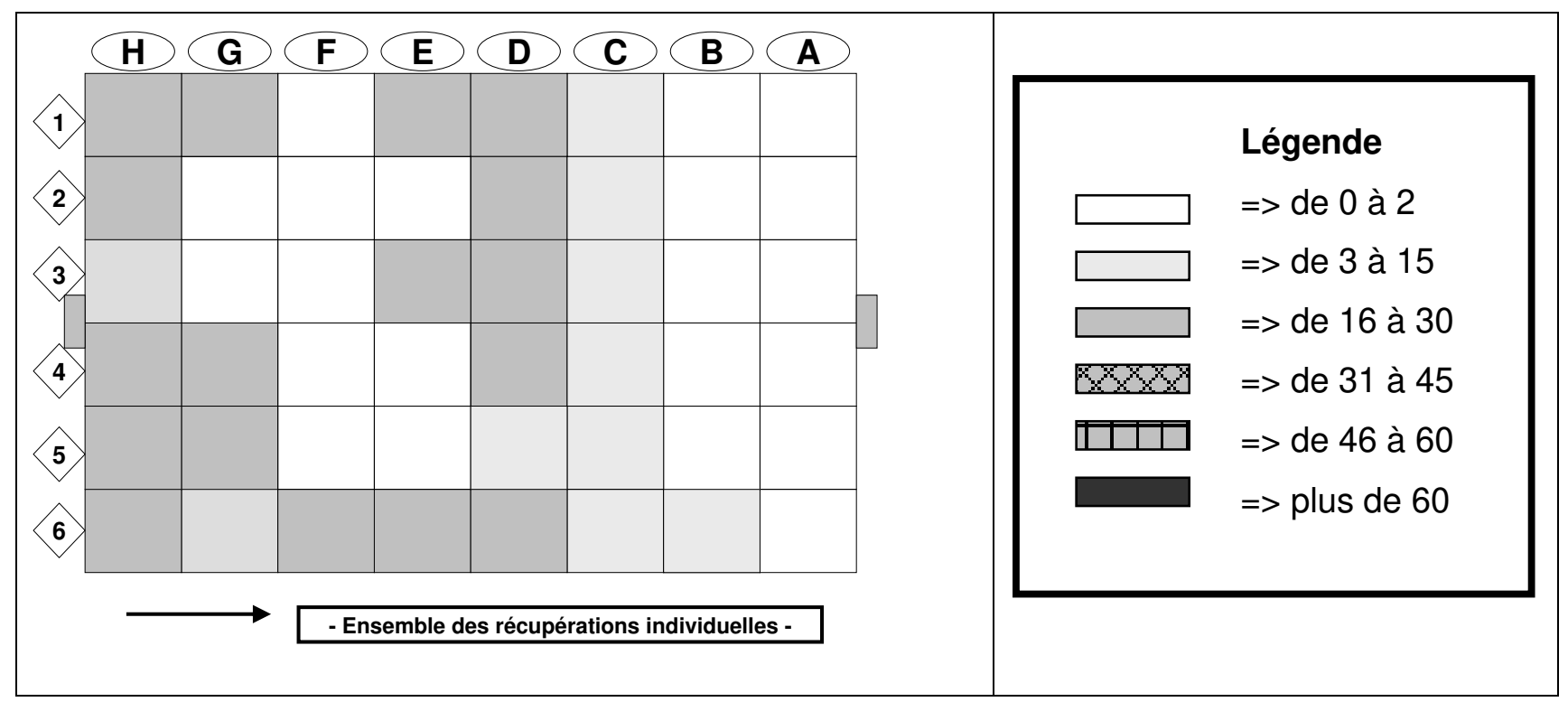

Figure 4. Répartition des récupérations individuelles

La notion de densité, mais surtout de harcèlement, pour les défenses hautes pourrait aussi être une explication. Cela demanderait une étude plus approfondie de ce cas 
spécifique, car lors des relances longues en contre-attaque la recherche des joueurs de pointe est contrariée par la présence des défenseurs en supériorité numérique. On remarque une efficacité défensive individuelle dans les cases $\mathrm{H}-1$ et $\mathrm{H}-6$, ce qui pourrait confirmer notre idée « d'enfermement ». Les effectifs relativement importants en $\mathrm{H}-4$ et $\mathrm{H}$ 5 correspondent à l'utilisation des passes longues en transversales des attaquants, venant du flanc gauche défensif et finissant leur trajectoire dans cette zone, au même titre que les « $R G$ » en coups de pied de but.

3. 4. Les " récupérations collectives »

Pour les « récupérations collectives » (figure 5), on distingue très nettement un bloc placé plus bas que le précédent et surtout réparti essentiellement de chaque côté de l'axe du terrain, avec une confirmation de la tendance sur le flanc gauche en F-2. Plus rapproché du but, le positionnement des récupérations collectives confirmerait l'idée que la densité, permise par le recentrage des joueurs en défense à l'approche du but, favorise la reconquête.

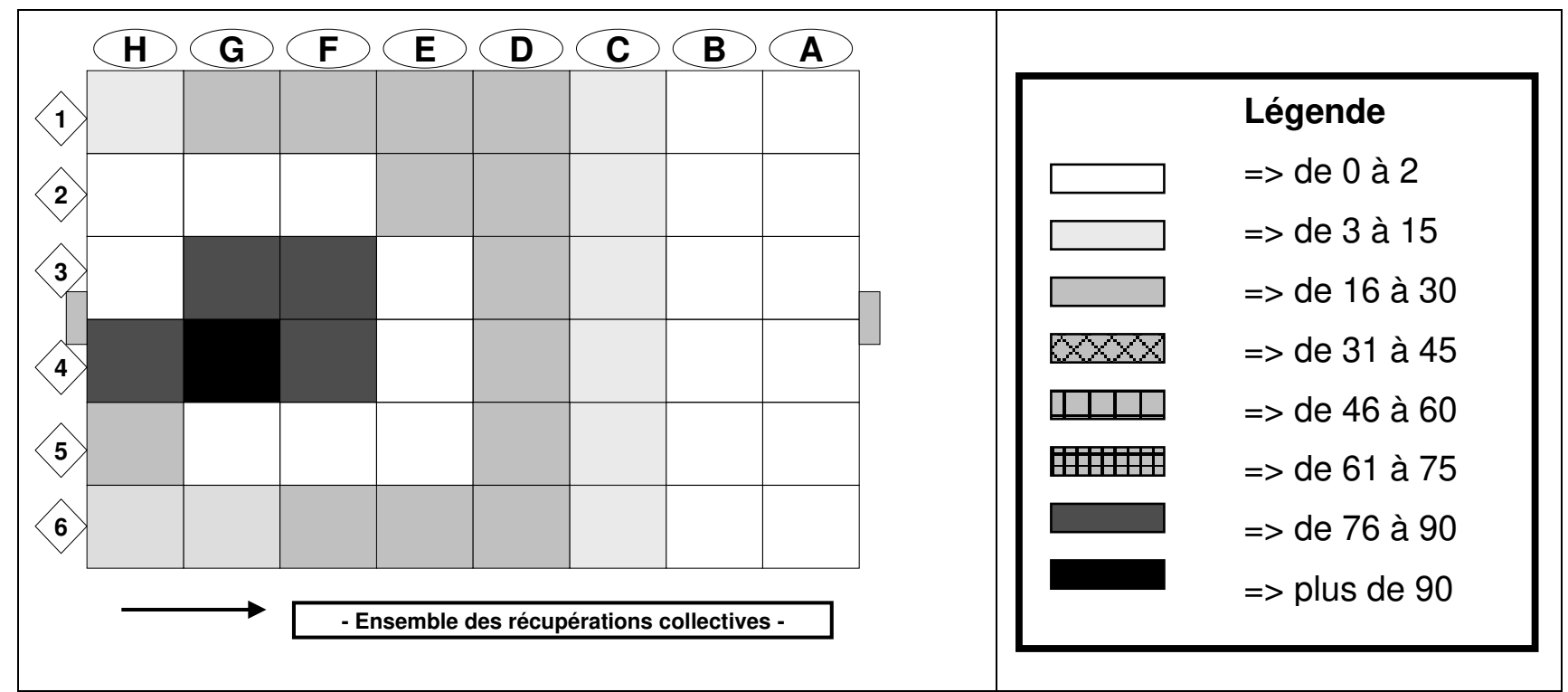

Figure 5. Répartition des récupérations collectives

Le grand nombre de récupérations en bande $E$ confirme nos propos précédents sur les gains de balles rendus possibles grâce à la supériorité numérique du dernier rideau défensif, hors gardien de but, lors du jeu en contre-attaque des adversaires. Nous avions remarqué que la zone $F$ devance légèrement la zone $G$ du point de vue de l'ensemble des récupérations collectives. Néanmoins ces récupérations collectives se trouvent beaucoup plus étalées sur la largeur en $F$ qu'en $G$ où nous avons un rapprochement de l'axe du terrain. 
3. 5. Les récupérations du gardien de but

Pour ce type de récupération, signalons la particularité du statut de ce joueur à qui l'on donne le pouvoir d'utiliser ses mains. II représente le dernier rempart et évolue essentiellement dans sa surface de réparation où aboutit normalement tout mouvement offensif adverse. Son intervention dans la profondeur se limite dans la majorité des cas à la zone $\mathrm{H}$, proche de son but, mais peut parfois s'étendre vers l'avant (zone G), surtout avec les " organisations défensives hautes " où le gardien de but peut être amené à sortir de sa surface de réparation pour assurer la couverture de ses partenaires. Sur les 346 ballons arrêtés, interceptés, repoussés ou récupérés par les gardiens de but, 324 le sont dans la zone $\mathrm{H}$ et 21 dans la zone $\mathrm{G}$, plus un en zone $\mathrm{F}$.

3. 6. Présentation des récupérations sur plusieurs zones

Nous rappelons qu'à chaque étape intervient un défenseur dont l'action se limite à un seul contact avec le ballon. Nous constatons que sur les 858 actions ayant cette particularité, 3 se sont soldées par une récupération réglementaire, 16 par une récupération individuelle et 839 , soit presque l'ensemble $(97,8 \%)$, par une récupération collective. Pour ce qui est de l'orientation prise par le ballon suite à la première intervention, notons que : 231 soit $26,9 \%$ suivent un décalage vers la droite ou la gauche, 418 soit $48,7 \%$ aboutissent suite à un renvoi vers l'avant et 209 soit $24,4 \%$ résultent d'un prolongement, ou du retour d'un joueur qui pousse le ballon, vers son propre but pour un de ses partenaires. Précisons que 34 , soit $4 \%$, de ces interventions ont impliqué trois joueurs situés dans des zones différentes ce qui en fait des situations exceptionnelles (environ $1 \%$ ) lorsqu'on les resitue dans les 3460 récupérations totales.

\section{Discussion}

L'analyse globale des récupérations laisse apparaître l'importance de gérer collectivement la reconquête du ballon. Aux "récupérations collectives ", qui représentent plus d'un tiers des récupérations, on pourrait ajouter une partie des "récupérations réglementaires " mais aussi des « récupérations individuelles».

En effet, si l'on observe les « modes d'actions » mis en œuvre, on constate que :

- pour les « récupérations réglementaires » on pourrait valider les touches, mais aussi les récupérations sanctionnant un hors-jeu comme résultant d'une récupération collective. Dans le premier cas, les limites du terrain constituent une contrainte apparentée à la présence d'un adversaire. Dans le second qui concerne le hors-jeu, la nécessité d'une organisation collective en amont ou de faire appel à l'intelligence du joueur dans certaines situations spécifiques confère à la loi 11 un statut de partenaire par défaut ; 
- pour les « récupérations individuelles », nous constatons que 32 \% d'entre-elles résultent d'une récupération d'un joueur seul ce qui implique au préalable une erreur adverse, une bonne lecture du jeu du défenseur qui agit suite à l'action de l'attaquant sous pression, ou bien l'intervention d'un premier défenseur.

Nous pourrions donc comptabiliser ces récupérations issues pour une part des « RR » et pour l'autre part des « $\mathrm{RI}$ » dans les « récupérations collectives » qui atteindraient alors les $59,3 \%$.

On pourrait même y ajouter une partie des «RR» suite à un coup de pied de but à l'exemple des touches, et une autre résultant des « $\mathrm{RI}$ »en conquête faisant suite à une intervention d'un partenaire au départ du ballon.

\section{Conclusion}

II ressort de notre étude qu'il existe réellement des endroits sur le terrain où la récupération du ballon est plus efficace en fonction de certains facteurs tels que : la densité des joueurs ; la réduction du pouvoir d'action des attaquants en utilisant les limites de l'espace de jeu ; les logiques d'accès à la cible qui ramènent inévitablement le ballon vers l'axe du terrain. Sur le plan topocinétique, les reconquêtes se situent plutôt sur le flanc droit de la défense car nous retrouvons les confrontations en conquête résultant du jeu transversal venant de la droite vers le centre pour les attaquants, mais aussi le retour vers l'axe des attaques orientées initialement sur la gauche, dès leur entrée dans la moitié offensive. C'est logiquement dans la bande $\mathrm{H}$, juste devant le but, dans une position axiale, que l'on trouve le plus de récupérations. Cela constitue la dernière partie de l'entonnoir dont le goulot est la cible.

II apparaît nettement que la reconquête du ballon est, quel que soit le type de récupération, pour une très grande majorité des cas, l'œuvre d'actions coordonnées, articulées entre plusieurs éléments du collectif. Même lorsqu'il s'agit d'utiliser les lois du jeu pour provoquer la faute, l'action du joueur directement concerné par le ballon s'inscrit dans une globalité assujettie aux placements des partenaires. Lorsqu'on constate, en première intention, qu'un seul défenseur est impliqué dans la récupération, la réussite de son action est très souvent dépendante du soutien ou de l'action préalable d'un partenaire. Pour qu'une « récupération collective » puisse avoir lieu il est nécessaire que plusieurs éléments entrent en action en même temps, souvent dans le même espace ou dans un environnement proche, et parfois même assez loin de l'affrontement direct. L'étude des « modes d'actions » pour chaque « type de récupération » montre bien la prégnance et la nécessité d'une action coordonnée pour être efficace dans la phase de récupération. Nous 
obtenons aussi, par inférence, des informations sur l'orientation majoritaire des mouvements offensifs. C'est la confirmation, à notre sens, que les équipes comportent plus de droitiers que de gauchers ce qui expliquerait cette orientation marquée. Cela pourrait aussi montrer que les joueurs capables de jouer des deux pieds auraient tendance à revenir sur le pied fort (droit) pour tirer ou achever leur action. Tous ces éléments s'inscrivent comme des sources d'informations précieuses pour l'intervention du formateur. D'autant plus, qu'au regard d'une étude parallèle, nous pouvons avancer l'idée que les types de récupérations découlent non seulement des logiques d'actions et des potentiels des joueurs présents sur le terrain, mais aussi des choix tactiques, stratégiques mis en œuvre par les équipes. Nous aurions pu élargir notre champ de connaissances si nous avions étendu notre observation à l'origine de l'action. Cela nous aurait permis d'analyser les systèmes socio-techniques mis en œuvre pour être efficace en défense.

\section{Bibliographie :}

Bonizzoni, L. \& Comucci, N. (1979). La tecnica calcistica - il calcio : come si impara. Guide dell'allenatore. Italia : Nardini Editore.

Bouthier, D. \& Durey, A. (1994). Technologie des Activités Physiques et Sportives. Revue Impulsion (1), (pp. 95-123). Paris : INRP.

Castanõ Gomez, C. M. (1999). Usted es un entrenador. Medellin : Universitad Cooperativa de Colombia.

Comucci, N. (1977). L'allenamento al gioco di squadra. FIGC Setore tecnico. Guide Dell'allenatore, Ilalia : Nardini Editore.

David, B., Bouthier, D., Marsenach, J., \& Durey, A. (1999). French research into the didactique and technology of physical activity and sport : an expanding new field. Instructionnal science, 17, 148-162.

Deleplace, R. (1979). Rugby de mouvement, rugby total. Paris : Revue EPS.

Dugrand, M. (1989). Football de la transparence à la complexité. Paris: Presses Universitaires de France.

Duprat, E. (1996). Technique et prise de décision : l'exemple du tacle en football. Diplôme d'Etude Approfondies, Université Paris XI, Orsay.

Duprat, E. (2005). Approche technologique de la récupération du ballon lors de la phase défensive en football, contribution à l'élaboration de contenus de formations innovants. Thèse de Doctorat, École Normale Supérieure de Cachan. 
Gréhaigne, J. F. (1989). Football de mouvement : vers une approche systémique du jeu. Thèse de Doctorat, Université de Dijon.

Gréhaigne, J. F. (1992). L'organisation du jeu en football. Paris : Actio.

Gréhaigne, J. F. \& Bouthier D. (1994). Analyse des évolutions entre deux configurations de jeu en football. Revue Sciences et motricité, 24, 44-52.

Gréhaigne, J. F., Marchal, D. \& Duprat, E. (2001). Regaining possession of the ball in the défense area in soccer. In W. Spinks, T. Reilly, \& A. Murphy (Eds). Revue Sciences and football, 4. London : Taylor and Francis.

Grosgeorges, B. (1990). Observation et entraînement en sports collectifs. Collection Entraînement. Paris : INSEP Publications.

Hedergott, K.H. (1976). Nouvel enseignement du football. Frankfurt, R.F.A. : Limpert Verlag.

Lemoine A. (2003). Etude de la production d'informations dans le cadre de la transmission instantanée du ballon au football. Thèse de Doctorat (non publiée). Université de Picardie « Jules Vernes»

Parlebas, P. \& Cyffers, B. (1992). Statistique appliquée aux Activités Physiques et Sportives. Collection Etudes et Formation. Paris : INSEP.

Sérafin, J. (1982). Les aspects tactiques dans le football de haut niveau. Mémoire de I'INSEP.

Téodorescu, L. (1965). Principes pour l'étude de la tactique commune aux jeux sportifs collectifs. Compte-rendu du Colloque international Sports Collectifs. Vichy: Revue de la S.I.E.R.E.P.S., 3, 29-40.

Téodorescu, L. (1977). Théorie et méthodologie des jeux sportifs. Collection Sport et progrès de l'homme. Paris : Les Editeurs Français Réunis.

Vigarello, G. (1988). Techniques d'hier... et d'aujourd'hui : une histoire culturelle du sport. Paris : R. Laffont.

Wallon, H. (1968). L'évolution psychologique de l'enfant. Paris : Armand Colin. 